\title{
Social Welfare Functions for Different Subgroup Utility Scales ${ }^{\star}$
}

\author{
Anna B. Khmelnitskaya ${ }^{\star \star}$ \\ $\mathrm{SPb}$ Institute for Economics and Mathematics Russian Academy of Sciences, \\ 1 Tchaikovsky St., 191187 St.Petersburg, Russia
}

\begin{abstract}
This paper characterizes social welfare functions for different scales of individual utility measurement in distinct population subgroups. Different combinations of ordinal, interval, ratio, and translation scales are studied. We consider situations when utility comparisons among subgroups of individuals by unit and/or zeropoint can or cannot be made, that is when subgroup utility scales are dependent or independent. We show that for combinations of independent subgroup utility scales every corresponding social welfare function is fully determined by the opinions of only one subgroup of individuals when there is not more than one subgroup with ratio or translation scale measurable utilities, otherwise it also might be determined by the union of subgroups corresponding to ratio and translation scales, and is in accord with the utility scales of the decisive coalition. We also investigate social welfare functions admissible given various combinations of interval scales with a common unit that combine individual utilities from different subgroups, or with a common zero that lead to existence of a dictatorial subgroup.
\end{abstract}

Keywords: Arrovian social choice, social welfare function, interpersonal utility comparisons.

\section{Introduction}

In Arrow's (1963) famous impossibility theorem, individual preferences are ordinally measurable and not interpersonally comparable. In a number of studies (Sen (1970), Roberts (1980a, b), d'Aspremont (1985), Yanovskaya (1988, 1989), Bossert and Weymark (1996)), more restrictive measurability-comparability assumptions have been proposed and investigated. These studies show that under different measurability-comparability conditions for individual preferences, nondictatorial social choice rules exist. However, in these studies, the measurement scales for individual preferences are assumed to be of the same type across the entire society.

* Support from the Russian Foundation for Basic Research (grant \#96-01-00170) and the European Community (grant \#INTAS-96-106) is gratefully acknowledged.

** I would like to express my sincere gratitude to Elena Yanovskaya, John Weymark, Kevin Roberts and Natalia Naumova for fruitful discussions and helpful suggestions. 
The goal of this paper is to study Arrovian social choice problems when individual preferences in disjoint subgroups of individuals are measured by different scale types, in other words, when separate subgroups of individuals admit different types of information. This situation is common in real decision making. A typical example is the partitioning of a human society into families which in turn consist of individuals. If an outsider is making the comparisons based on reports from individuals, it is reasonable to suppose that the kind of information available within and between families will be different in general. Indeed, the kinds of utility comparisons that can be made within a family cannot be made between people who do not know each other.

We adopt the welfarist approach to social choice and assume that only individual utilities matter for ranking a feasible set of social alternatives. In an Arrovian social choice problem, this is implied by Pareto Indifference and Independence of Irrelevant Alternatives; the social choice rule can be equivalently described either in terms of a social welfare ordering - a social ordering of the admissible profiles of individual utilities (admissibility is understood as the satisfaction of several a priori appealing conditions), or in terms of a social welfare function - a function that represents a social welfare ordering and measures social welfare. Various assumptions concerning the measurability and interpersonal comparability of utility can be formalized by partitioning the set of feasible individual profiles of utility functions and requiring the social welfare ordering to be constant over a cell of the partition.

In Sect. 2, we introduce basic definitions and notation and provide a formal statement of the problem. In Sect. 3, various combinations of independent subgroup scales that lead to the existence of a decisive coalition are considered. It is proved that if there is not more than one subgroup of individuals with ratio or translation scale measurable utilities, then the decisive coalition is equal to one of the subgroups, otherwise the decisive coalition may coincide with the union of the latter subgroups. Sect. 4 studies different combinations of dependent interval subgroup scales. Sect. 5 provides some concluding remarks. Some proofs are given in Appendix.

\section{The framework}

Consider a society consisting of a finite set $N=\{1, \ldots, n\}$ of $n \geq 2$ individuals. Let $X$ be a set of at least three alternatives and let $\mathcal{R}$ denote the set of all possible preference orderings over $X$. The members of $\mathcal{R}$ are assumed to be weak orders, i.e., complete, reflexive and transitive binary relations. A social choice problem is a triple $\left\langle X, N,\left\{R_{i}\right\}_{i \in N}\right\rangle$, where $\left\{R_{i}\right\}_{i \in N}$ is a profile of individual preferences $R_{i} \in \mathcal{R}, i \in N$. To introduce measurability/comparability assumptions, we consider individual preferences represented as individual utilities, which may be interpreted as measurements of these preferences. So, let $U$ be the set of all real-valued functions defined on $X \times N$ : for any $u \in U$, let $u(x, i)$ denote the $i$ th individual utility at the alternative $x \in X$. A social welfare functional is a mapping $f: \mathcal{D} \rightarrow \mathcal{R}$, where $\mathcal{D} \subseteq U$ is the domain of $f$. We assume that $f$ satisfies 
three welfarism axioms:

Unrestricted Domain. $\mathcal{D}=U$, i.e., $f$ is defined for all $u \in U$.

Independence of Irrelevant Alternatives. For any $u, u^{\prime} \in \mathcal{D}$ and $A \subseteq X$, if $u(x, i)=u^{\prime}(x, i)$ for all $x \in A$ and $i \in N$, then $R: A=R^{\prime}: A$, where $R=f(u)$ and $R^{\prime}=f\left(u^{\prime}\right)$. ( $R: A$ denotes the restriction of $R$ to $A \subseteq X$.)

Pareto Indifference. For any pair $x, y \in X$ and for all $u \in \mathcal{D}$, if $u(x, i)=u(y, i)$ for all $i \in N$, then $x I y$, where $I$ denotes the indifference relation corresponding to $R=f(u)$.

According to the welfarism theorem (D'Aspremont and Gevers (1977), and Hammond (1979)), these three axioms ensure that only individual utilities matter when ranking social alternatives, so any vector $u=\left(u_{1}, \ldots, u_{n}\right)$ in the $n$ dimensional Euclidian space $\mathbb{R}^{n}$ can be considered as a profile of individual utilities for the society $N$; here $u_{i}$ is the utility of $i$ th individual. From this perspective, a solution to a social choice problem can be regarded as a social welfare ordering (SWO), which is a weak order $R^{*}$ on $\mathbb{R}^{n}$, the set of possible profiles of utility vectors. We assume that $R^{*}$ also satisfies

Weak Pareto (WP). For all $u, v \in \mathbb{R}^{n}$, if $u_{i}>v_{i}$ for all $i \in N$, then $u P^{*} v$, where $P^{*}$ denotes the strict preference relation corresponding to $R^{*}$.

A function $W: \mathbb{R}^{n} \rightarrow \mathbb{R}^{1}$ represents the $\mathrm{SWO} R^{*}$ if for all $u, v \in \mathbb{R}^{n}$

$$
u R^{*} v \Longleftrightarrow W(u) \geq W(v) .
$$

The representation $W$ is called a social welfare function (SWF). By WP, any SWF $W$ is strictly increasing, i.e., for all $u, v \in \mathbb{R}^{n}$

$$
u \gg v \Longrightarrow W(u)>W(v) .
$$

We impose one more restriction on an SWO $R^{*}$. We require $R^{*}$ to be continuous.

Continuity (C). For all $u \in \mathbb{R}^{n}$, the sets $\left\{v \in \mathbb{R}^{n} \mid v R^{*} u\right\}$ and $\left\{v \in \mathbb{R}^{n} \mid u R^{*} v\right\}$ are closed in $\mathbb{R}^{n}$.

Continuity guarantees the existence of a continuous SWF (see Debreu (1954)).

In what follows by $D_{n}$, we denote the diagonal of $\mathbb{R}^{n}$. Let for any real $c, c_{N}$ be a vector in $\mathbb{R}^{n}$ with all components equal to $c$ and let $\gamma(c)=\left\{u \in \mathbb{R}^{n} \mid W(u)=c\right\}$ 
be a $c$-level surface of the SWF $W$; obviously, for every $u \in \mathbb{R}^{n}, \gamma(W(u))$ is a level surface of $W$ containing $u$.

Because of continuity and strict monotonicity of all SWF, every level surface of any SWF intersects a diagonal $D_{n}$ of $\mathbb{R}^{n}$ and, moreover, this intersection is a singleton. So, a natural scale for an SWF arises: since every SWF $W$ is defined up to a strictly increasing transform, then, without loss of generality, it may be assumed that for any $u \in \mathbb{R}^{n}, W(u)=c$, with $c$ defined by the equality $\gamma(W(u)) \cap D_{n}=\left\{c_{N}\right\}$.

In the classic case of Arrow, utilities are ordinally measurable and interpersonally non-comparable. More generally, within the SWO framework, the degree of measurability and comparability of utility inside the society $N$ can be specified by a class of invariance transforms $\Phi$, where each transform $\phi \in \Phi$ is a list of functions $\phi=\left\{\phi_{i}\right\}_{i \in N}, \phi_{i}: \mathbb{R}^{1} \rightarrow \mathbb{R}^{1}$, with the property that for all $u, v \in \mathbb{R}^{n}$

$$
u R^{*} v \Longleftrightarrow(\phi u) R^{*}(\phi v)
$$

where $\phi u=\left\{\phi_{i} u_{i}\right\}_{i \in N}$. In what follows, if we wish to specify that the transforms of a class $\Phi$ apply to a particular society $N$, we use the notation $\Phi_{N}$; when there is no ambiguity, the index $N$ will be omitted.

For a class $\Phi$ to be a scale in the sense of the standard theory of measurement it has to satisfy the stronger condition of being a group (see Phanzagl, (1971)). Different scale types for individual utility measurement have been examined in the literature (Roberts (1980b), d'Aspremont (1985), Bossert and Weymark (1996)). Next we list the invariance classes to be considered here, the most of them are scales.

Ordinal Measurability (OM).$\phi \in \Phi$ iff $\phi$ is a list of any strictly increasing transforms $\phi_{i}, i \in N$.

Ordinal Measurability and Full Comparability (OFC). $\phi \in \Phi$ iff $\phi$ is a list of identical strictly increasing transforms, i.e., for any real $t$ and all $i \in N$, $\phi_{i}(t)=\phi_{0}(t)$, where $\phi_{0}$ is a strictly increasing function independent of $i$.

Cardinal Measurability (CM). $\phi \in \Phi$ iff $\phi$ is a list of any strictly increasing affine transforms, i.e., for any real $t$ and all $i \in N, \phi_{i}(t)=\alpha_{i}+\beta_{i} t$ for some real $\alpha_{i}$ and real $\beta_{i}>0$.

Cardinal Measurability and Origin Comparability (COC). $\phi \in \Phi$ iff $\phi$ is a list of strictly increasing affine transforms with a common constant term, i.e., for any real $t$ and all $i \in N, \phi_{i}(t)=\alpha+\beta_{i} t$ for some real $\alpha$ and $\beta_{i}>0$ with $\alpha$ independent of $i$.

Notice that a class of COC transforms is not a scale since it does not possess the group property. All the others are scales. 
Cardinal Measurability and Unit Comparability (CUC). $\phi \in \Phi$ iff $\phi$ is a list of strictly increasing affine transforms with a common unit, i.e., for any real $t$ and all $i \in N, \phi_{i}(t)=\alpha_{i}+\beta t$ for some real $\alpha_{i}$ and $\beta>0$ with $\beta$ independent of $i$.

Cardinal Measurability and Full Comparability (CFC). $\phi \in \Phi$ iff $\phi$ is a list of identical strictly increasing affine transforms, i.e., for any real $t$ and all $i \in N \quad \phi_{i}(t)=\alpha+\beta t$ for some real $\alpha$ and $\beta>0$, both independent of $i$.

Ratio-Scale Measurability (RSM). $\phi \in \Phi$ iff $\phi$ is a list of strictly increasing affine transforms with zero constant term, i.e., for any real $t$ and all $i \in N$, $\phi_{i}(t)=\beta_{i} t$ for some $\beta_{i}>0$.

Ratio-Scale Measurability and Full Comparability (RSF). $\phi \in \Phi$ iff $\phi$ is a list of identical strictly increasing affine transforms with zero constant term, i.e., for any real $t$ and all $i \in N, \phi_{i}(t)=\beta t$ for some $\beta>0$ independent of $i$.

Translation-Scale Measurability (TSM). $\phi \in \Phi$ iff $\phi$ is a list of strictly increasing affine transforms whose unit term is 1, i.e., for any real $t$ and all $i \in N, \phi_{i}(t)=\alpha_{i}+t$ for some real $\alpha_{i}$.

Translation-Scale Measurability and Full Comparability (TSF). $\phi \in \Phi$ iff $\phi$ is a list of identical strictly increasing affine transforms whose unit term is 1 , i.e., for any real $t$ and all $i \in N, \phi_{i}(t)=\alpha+t$ for some real $\alpha$ independent of $i$.

The considered invariant transform classes can be naturally split into two types - one-parameter and two-parameter. Transform classes of the second type are those that contain two-parameter affine transforms (OM, OFC, CM, COC, CUC, CFC), and of the first type that admit affine transforms with only one free parameter, i.e., either with a zero constant term (RSM, RSF) or with an a priori fixed unit equal to one (TSM, TSF).

Under the conditions imposed, the Arrovian social choice problem in the informational environment introduced by an invariance class $\Phi$ can be equivalently described in terms of SWF $W$ which

1 ) is a continuous real-valued function defined on $\mathbb{R}^{n}$ such that $W\left(c_{N}\right)=c$ for any real c;

2) is nondecreasing ${ }^{1}$, i.e., for all $u, v \in \mathbb{R}^{n}$,

$$
u \geq v \Longrightarrow W(u) \geq W(v)
$$

\footnotetext{
${ }^{1}$ This holds because $W$ is continuous and strictly increasing.
} 
3) is invariant under invariance transforms of class $\Phi$, i.e., for any $\phi \in \Phi$ and for all $u, v \in \mathbb{R}^{n}$,

$$
W(u) \geq W(v) \Longleftrightarrow W(\phi u) \geq W(\phi v) .
$$

The main concern of this paper is the situation where the entire society $N$ is partitioned into $m$ disjoint subgroups of individuals, i.e., $N=N_{1} \cup N_{2} \cup \cdots \cup N_{m}$ with $N_{i} \cap N_{j}=\emptyset$ for $i \neq j$. It is assumed that an SWF $W$ defined on $\mathbb{R}^{n}$ for different subgroups of variables indexed by $N_{k}, k \in\{1, \ldots, m\}$, may admit invariance transforms of different invariance classes $\Phi_{N_{k}}$, which amounts to $W$ being invariant under transforms of a class $\Phi_{N}$ such that $\Phi_{N}=\left\{\Phi_{N_{k}}\right\}_{k=1}^{m}$, i.e., for every $\phi \in \Phi_{N}$ for all $k \in\{1, \ldots, m\}, \phi_{N_{k}}=\left\{\phi_{i}\right\}_{i \in N_{k}} \in \Phi_{N_{k}}$. In other words $\Phi_{N}$ is the Cartesian product of the subgroup classes of transforms $\Phi_{N_{k}}$. It is worth noting that the class $\Phi_{N}$ has to satisfy condition (1); however, in general, even if all invariant classes $\Phi_{N_{k}}$ are scales, $\Phi_{N}$ may not be a scale: the condition of being a group may no longer hold. For example, any combination of CFC scales with a common zero is not a scale.

To complete this section we introduce some additional notation. By $n_{k}$ we denote the cardinality of $N_{k}$. It is obvious that $\sum_{k=1}^{m} n_{k}=n$. For any $u \in \mathbb{R}^{n}$ and all $k \in\{1, \ldots, m\}$, let $u_{N_{k}}$ be a subvector of $u$ that belongs to $\mathbb{R}^{n_{k}}$ and that is composed of components $u_{i}$ with $i \in N_{k}$. $\mathbb{R}^{N_{k}}$ is the coordinate subspace of $\mathbb{R}^{n}$ induced by coordinates with indices from $N_{k}$, i.e.

$$
\mathbb{R}^{N_{k}}=\left\{v \in \mathbb{R}^{n} \mid v_{i}=0, i \notin N_{k}\right\} .
$$

Denote by

$$
D_{N_{k}}=\left\{u \in \mathbb{R}^{N_{k}} \mid u_{i}=u_{j}, i, j \in N_{k}, \quad \& \quad u_{i}=0, i \notin N_{k}\right\}
$$

the diagonal of the coordinate subspace $\mathbb{R}^{N_{k}} . L^{D}$ is the subspace of $\mathbb{R}^{n}$ spanned by the diagonals $D_{N_{k}}, k \in\{1, \ldots, m\}$. It is easy to see that every $u \in L^{D}$, $u=\left\{u_{i}\right\}_{i \in N}$, has the form $u_{i}=v_{k}$ for all $i \in N_{k}$ under some $v=v(u) \in \mathbb{R}^{m}$, i.e., all variables in $L^{D}$ indexed by the same subgroup of indices have the same value.

For any vector $u \in \mathbb{R}^{n}$, let $\hat{u}(\check{u})$ be the vector in $\mathbb{R}^{n}$ with all components equal to the largest (smallest) component of $u$. As usual, we designate the mean value of a vector $u \in \mathbb{R}^{n}$ by $\bar{u}$, i.e., $\bar{u}=\left(\sum_{i=1}^{n} u_{i}\right) / n$. The nonnegative and positive orthants in $\mathbb{R}^{n}$ are denoted by $\mathbb{R}_{+}^{n}$ and $\mathbb{R}_{++}^{n}$, respectively, i.e.,

$$
\begin{gathered}
\mathbb{R}_{+}^{n}=\left\{u \in \mathbb{R}^{n} \mid u_{i} \geq 0, i \in N, \quad \& \quad u \neq \mathbf{0}\right\}, \\
\mathbb{R}_{++}^{n}=\left\{u \in \mathbb{R}^{n} \mid u_{i}>0, i \in N\right\} .
\end{gathered}
$$

In what follows, we also borrow the notion of a fan defined in Bossert and Weymark (1996). For a vector $u \in \mathbb{R}^{n}$, the fan generated by $u$ is

$$
Y(u)=\left\{u^{\prime} \in \mathbb{R}^{n} \mid u^{\prime}=\theta 1_{n}+\lambda u, \theta \in \mathbb{R}, \lambda \in \mathbb{R}_{+}\right\} .
$$


A subset $Y$ of $\mathbb{R}^{n}$ is a fan if it is a fan generated by some $u \in \mathbb{R}^{n}$.

In what follows, we use the notation $N_{k}^{(2)}$ if we need to emphasize that an SWF with respect to variables indexed by $N_{k}$ is invariant under two-parameter type transforms, and we denote by $N^{(1)}$ a union of all subgroups $N_{k}$ corresponding to one-parameter type scales. Any subgroup of variables $N_{k}^{(2)}$ or $N^{(1)}$ are called a two-parameter or one-parameter type subgroup, respectively. When it is necessary to indicate that an SWF is invariant with respect to the subgroup of variables indexed by $N_{k}$ under $\Phi$ transforms, we use the notation $N_{k}(\Phi)$. If it is desirable to stress what class of invariance transforms is admissible for an SWF, we use the designation $\operatorname{SWF}(\Phi)$, and an $\operatorname{SWF}(\Phi)$ characterization, we call an $\Phi$-characterization.

\section{Independent subgroup scales}

\section{Existence of a decisive coalition.}

First, we examine situations with mutually independent subgroup scales for which a decisive coalition equal to either a two-parameter type (OM, OFC, CM, CUC, CFC) subgroup of individuals or to the union of all one-parameter type (RSM, RSF, TSM, TSF) subgroups must exist.

The subgroup classes of transforms $\Phi_{N_{k}}, k \in\{1, \ldots, m\}$, are mutually independent if, for any two distinct $k_{1}, k_{2} \in\{1, \ldots, m\}$, for every $i \in N_{k_{1}}$, and for every $j \in N_{k_{2}}$, the transforms $\phi_{i} \in \Phi_{N_{k_{1}}}$ and $\phi_{j} \in \Phi_{N_{k_{2}}}$ are independent, i.e., if the transforms that apply to the variables of one subgroup are independent of the transforms that apply to any other subgroup. Mutual Independence preserves the group property and guarantees $\Phi_{N}$ to be the direct product of groups $\Phi_{N_{k}}$ when each of the $\Phi_{N_{k}}$ is a group, i.e., it guarantees $\Phi_{N}$ to be a scale if all $\Phi_{N_{k}}$ are scales. It should be stressed that Mutual Independence is a property of the set of subgroup classes of transforms $\left\{\Phi_{N_{k}}\right\}_{k=1}^{m}$, not of individual transforms within these classes.

Theorem 3.1. Let $\left\{N_{k}\right\}_{k=1}^{m}$ be a partition of $N$ and let $W$ be a continuous, nondecreasing real-valued function defined on $\mathbb{R}^{n}$, that is invariant with respect to variables indexed by $N_{k}$ under any one of the invariance transform classes listed above. Invariance transforms proper to distinct subgroups of indices $N_{k}$ are assumed to be mutually independent. Then, either there exists a unique integer $k \in\{1, \ldots, m\}$ corresponding to a two-parameter type subgroup of variables indexed by $N_{k}^{(2)}$ such that the function $W$ has the form

$$
W(u)=W\left(u_{N_{k}^{(2)}}\right)
$$

for all $u \in \mathbb{R}^{n}$ or, for all $u \in \mathbb{R}^{n}, W$ is independent of all two-parameter type variables and depends only on one-parameter type variables, i.e., $W$ has the form

$$
W(u)=W\left(u_{N^{(1)}}\right) .
$$


Moreover, $W$ is fully characterized in accordance with the invariance transforms admissible to the subset of variables it determined by.

Theorem 3.1 shows that with mutually independent subgroup scales, every SWF is either determined by only one subgroup of two-parameter type variables, or is completely independent of all such variables and is fully determined by the one-parameter subgroups. Theorem 3.1 allows us to construct an SWF characterization for various combinations of arbitrary independent subgroup utility scales on the basis of well-known results for social choice problems with the same measurement scales of individual utilities for the entire society and the characterization results for combinations of only one-parameter type RSM, RSF, TSM, and TSF scales which are discussed later (see Theorem 3.4 and Theorem 3.5 below). For example, if in any one subgroup of individuals, utilities are measured in $\mathrm{OM}$ or $\mathrm{CM}$ scales, then either a dictator from this subgroup exists or the SWF is completely independent of this subgroup's utilities and is fully determined by the rest of the society in accordance with their utility scales.

A particular case of independent CUC scales for disjoint subgroups was investigated by Plata-Pérez (1994). Various combinations of mutually independent OM, OFC, CM, CUC, and CFC scales, i.e. only combinations of mutually independent two-parameter type scales, were studied in Khmelnitskaya (1996, 1999)), and Khmelnitskaya and Weymark (2000), who employed two different proof techniques. Our proof strategy for the general case is similar to that developed in Khmelnitskaya and Weymark (2000).

To prove Theorem 3.1, we employ the following lemma.

Lemma 3.2. Let $W$ be a continuous, nondecreasing real-valued function defined on $\mathbb{R}^{n}$. Suppose that $W$ is invariant with respect to some variable $u_{i_{0}}, i_{0} \in N$, under strictly increasing two-parameter affine transforms and with respect to the other variables under any one of the invariance transform classes listed above possibly different for different subgroups of variables. Then, either the function $W$ has the form

$$
W(u)=W\left(u_{i_{0}}\right)
$$

for all $u \in \mathbb{R}^{n}$ or, for all $u \in \mathbb{R}^{n}, W$ is independent of $u_{i_{0}}$ and has the form

$$
W(u)=W\left(u_{N \backslash\left\{i_{0}\right\}}\right) .
$$

The proofs of Lemma 3.2 and Theorem 3.1 are given in Appendix.

\section{Combinations of ratio and translation scales.}

Now we restrict consideration to different combinations of independent oneparameter type RSM, RSF, TSM, and TSF subgroup scales that, in general, do not give rise to only dictatorial subgroup characterization for an SWF. Note, that combinations of different RSM subgroups do not require a separate study, since this hypothesis is equivalent to supposing RSM invariance for the joint subgroup. It should be noticed also that a few singleton RSF subgroups amounts to the 
condition of RSM invariance for the union of them. Therefore, it is enough to consider only combinations of RSF subgroups. Similarly, we can easily see that it suffices to study only combinations of TSF subgroups instead of different combinations of TSM and TSF ones. Next, it is straightforward to check that if we replace all TSM and TSF variables $u_{i}, i \in N_{T}=\cup_{k \in \overline{1, m}} N_{k}$ (TSM or TSF), $u_{N_{T}} \in \mathbb{R}^{n_{N_{T}}}$, by the variables $v_{i}=\exp \left(u_{i}\right)$, then $v_{N_{T}} \in \mathbb{R}_{++}^{n_{N_{T}}}$ and the variables $v_{i}, i \in N_{T}$, turn out to be RSM or RSF variables, respectively.

In what follows we relay on the next general remark.

Remark 3.3. Suppose we examine a social choice problem for the society $N$ in the informational environment prescribed by the invariance class $\Phi$ (which, in particular, may be a certain combination of subgroup scales). Assume also that there are two other scales $\Phi^{\prime}$ and $\Phi^{\prime \prime}$ for which we know SWF characterizations. Suppose moreover, that $\Phi^{\prime} \subset \Phi \subset \Phi^{\prime \prime}$, and assume that there exists a subset $L^{\Phi} \subset \mathbb{R}^{n}, \operatorname{dim} L^{\Phi}=m$, such that for any $W \in \operatorname{SWF}(\Phi)$, the restriction $\left.W\right|_{L^{\Phi}}$ to $L^{\Phi}$ can be identified with a real-valued function $W^{*}$ on $\mathbb{R}^{m}$ that admits invariance transforms from the class $\Phi^{\prime \prime}$. Then we can state that an $\operatorname{SWF}(\Phi)$ possesses the $\Phi^{\prime}$-characterization that narrows to the $\Phi^{\prime \prime}$-characterization on $L^{\Phi}$. Furthermore, if $\Phi$ is a certain combination of subgroup scales, i.e., $N$ is partitioned into $m$ subgroups $\left\{N_{k}\right\}_{k=1}^{m}$ and $\Phi=\Phi_{N}=\left\{\Phi_{N_{k}}\right\}_{k=1}^{m}$, then, it is not difficult to see, that for every $k \in\{1, \ldots, m\}$ and for any fixed $\hat{u}_{N \backslash N_{k}} \in \mathbb{R}^{N \backslash N_{k}}$, the $\Phi^{\prime}$-characterization of every $\operatorname{SWF}\left(\Phi_{N}\right)$, considered as a function on $\mathbb{R}^{n_{k}}$, has to be consistent with the $\Phi_{N_{k}}$-characterization as well.

Now, let $\Phi$ be a combination of RSF subgroup scales, possibly different for different $k$. Then $\operatorname{RSF} \subset \Phi \subset \mathrm{RSM}$ and for any $\operatorname{SWF}(\Phi) W$, its restriction $\left.W\right|_{L^{D}}$ to $L^{D}$ can be identified with a real-valued function $W^{*}$ defined on $\mathbb{R}^{m}$ that is an SWF(RSM). Therefore, because of Remark 3.3 and applying the results of Kaneko and Nakamura (1979), and of Tsui and Weymark (1997) for RSMcharacterization combined with the SWF characterization under RSF invariance (see Roberts (1980b)) we arrive at

Theorem 3.4. Let $\left\{N_{k}\right\}_{k=1}^{m_{R}+m_{T}}$ be a partition of $N, n_{R}=\sum_{k=1}^{m_{R}} n_{k}, n_{T}=$ $\sum_{k=m_{R}+1}^{m_{R}+m_{T}} n_{k}$, and let $W$ be a continuous, nondecreasing real-valued function defined on $\mathbb{R}_{++}^{n_{R}} \times \mathbb{R}^{n_{\mathrm{T}}}$ (resp. $\mathbb{R}_{+}^{n_{R}} \times \mathbb{R}^{n_{\mathrm{T}}}$ ), that is invariant with respect to variables indexed by $N_{k}$ under $R S F$ transforms for $k \in\left\{1, \ldots, m_{R}\right\}$, and under TSF transforms for $k \in\left\{m_{R}+1, \ldots, m_{R}+m_{T}\right\}$. Invariance transforms proper to distinct subgroups of indices $N_{k}$ are assumed to be mutually independent. Then

1) the function $W\left(u_{N_{k}}, \tilde{u}_{N \backslash N_{k}}\right)$ for a fixed $\tilde{u}_{N \backslash N_{k}} \in \mathbb{R}^{n-n_{k}}$ considered as a function on $\mathbb{R}^{n_{k}}$, is homothetic on $\mathbb{R}_{++}^{n_{k}}$ (resp. $\mathbb{R}_{+}^{n_{k}}$ ) for $k \in\left\{1, \ldots, m_{R}\right\}$, and is translatable on $\mathbb{R}^{n_{k}}$ for $k \in\left\{m_{R}+1, \ldots, m_{R}+m_{T}\right\}$;

2) the function $W$ restricted to the subspace $L_{++}^{D_{R}} \times L^{D_{T}}\left(\text { resp. } L_{+}^{D_{R}} \times L^{D_{T}}\right)^{2}$, i.e., for all $u=\left\{u_{i}\right\}_{i \in N} \in \mathbb{R}^{n}$ such that for every $i \in N$ and for all $k \in$ $\left\{1, \ldots, m_{R}+m_{T}\right\}$ if $i \in N_{k}$, then $u_{i}=v_{k}$ for a certain $v=v(u) \in \mathbb{R}_{++}^{m_{R}} \times \mathbb{R}^{m_{T}}$

$\left.\overline{{ }^{2} L_{++}^{D}(\text { resp. }} L_{+}^{D}\right)$ is defined analogously to $L^{D}$ replacing $\mathbb{R}^{n}$ by $\mathbb{R}_{++}^{n}\left(\right.$ resp. $\left.\mathbb{R}_{+}^{n}\right)$. 
(resp. $\mathbb{R}_{+}^{m_{R}} \times \mathbb{R}^{m_{T}}$ ), has the form (up to a strictly increasing transform)

$$
W(u)=\sum_{k=1}^{m_{R}} \alpha_{k} \ln \left[v(u)_{k}\right]+\sum_{k=m_{R}+1}^{m_{R}+m_{T}} \alpha_{k} v(u)_{k}
$$

with $\alpha=\left\{\alpha_{k}\right\}_{k=1}^{m_{R}+m_{T}} \in \mathbb{R}_{+}^{m_{R}+m_{T}}$.

Tsui and Weymark (1997) show that under RSM invariance the only SWF defined on all of $\mathbb{R}^{n}$ is a strongly dictatorial one. Thus, employing the similar arguments as before, we obtain

Theorem 3.5. Let $\left\{N_{k}\right\}_{k=1}^{m_{R}+m_{T}}$ be a partition of $N$ and let $W$ be a continuous, nondecreasing real-valued function defined on $\mathbb{R}^{n}$, that is invariant with respect to variables indexed by $N_{k}$ under $R S F$ transforms for $k \in\left\{1, \ldots, m_{R}\right\}$, and under TSF transforms for $k \in\left\{m_{R}+1, \ldots, m_{R}+m_{T}\right\}$. Invariance transforms proper to distinct subgroups of indices $N_{k}$ are assumed to be mutually independent. Then, either there exists a unique integer $k \in\left\{1, \ldots, m_{R}\right\}$ such that the function $W$ has the form

$$
W(u)=W\left(u_{N_{k}}\right)
$$

for all $u \in \mathbb{R}^{n}$ and is homothetic on $\mathbb{R}^{n_{k}}$ or, for all $u \in \mathbb{R}^{n}, W$ depends only on variables indexed by $N_{T}=\cup_{k=1}^{m} N_{k}(\mathrm{TSF})$, i.e.,

$$
W(u)=W\left(u_{N_{T}}\right)
$$

for every $k \in\left\{m_{R}+1, \ldots, m_{R}+m_{T}\right\}, W$ is translatable on $\mathbb{R}^{n_{k}}$; and on $L^{D_{T}}$, i.e., for all $u=\left\{u_{i}\right\}_{i \in N_{T}} \in \mathbb{R}^{n_{T}}$ such that for every $i \in N_{T}$ and for $k \in\left\{m_{R}+1, \ldots, m_{R}+m_{T}\right\}$ if $i \in N_{k}(\mathrm{TSF})$, then $u_{i}=v_{k}$ for a certain $v=$ $v(u) \in \mathbb{R}^{m_{T}}, W$ has the form

$$
W(u)=\sum_{k=1}^{m_{T}} \alpha_{k} v(u)_{k}
$$

with $\alpha=\left\{\alpha_{k}\right\}_{k=1}^{m_{T}} \in \mathbb{R}_{+}^{m_{T}}$.

\section{Interrelations among subgroup scales}

\section{Interval scales with a common unit.}

To obtain SWF characterization for various combinations of interval scales with a common for the entire society unit we exploit again the observation done in Remark 3.3.

Let $\Phi$ be a combination of CFC subgroup scales, possibly different for different $k$ but with the same unit for all $k$. Then CFC $\subset \Phi \subset \mathrm{CUC}$ and for any $\operatorname{SWF}(\Phi) W$, its restriction $\left.W\right|_{L^{D}}$ to $L^{D}$ can be identified with a real-valued function $W^{*}$ defined on $\mathbb{R}^{m}$ that is an $\mathrm{SWF}(\mathrm{CUC})$. Therefore, utilizing the SWF characterization under CUC and CFC invariance (Roberts (1980b), Bossert and Weymark (1996)), we may state: 
Theorem 4.1. Let $\left\{N_{k}\right\}_{k=1}^{m}$ be a partition of $N$ and let $W$ be a continuous, nondecreasing real-valued function defined on $\mathbb{R}^{n}$, that is invariant with respect to variables indexed by $N_{k}$ under CFC transforms that may be different for different $k$ but that have the same common unit. Then for all $u \in \mathbb{R}^{n}, W$ has the form

$$
W(u)=\bar{u}+\theta(u-\bar{u})
$$

under some continuous and homogeneous of the first degree function $\theta: \mathbb{R}^{n} \rightarrow$ $\mathbb{R}^{1}$, and, moreover,

1) on any fan $Y$ in $\mathbb{R}^{n}$ and on a diagonal subspace $L^{D}, W$ is linear with semipositive coefficients which, in general, depend on $Y$ or $L^{D}$ respectively;

2) for every $k \in\{1, \ldots, m\}$ and any fixed $\tilde{u}_{N \backslash N_{k}} \in \mathbb{R}^{n-n_{k}}$, the function $W\left(u_{N_{k}}, \tilde{u}_{N \backslash N_{k}}\right)$ considered as a function on $\mathbb{R}^{n_{k}}$, on every fan $Y$ in $\mathbb{R}^{n_{k}}$ is linear with semipositive coefficients depending, in general, on $Y$.

Theorem 4.1 is tantamount to every level surface of $W$ flattening on each fan $Y$ in $\mathbb{R}^{n}$, on each cylinder $Y^{\prime}+u_{N \backslash N_{k}}$ where $Y^{\prime}$ is a fan in $\mathbb{R}^{n_{k}}$ and $u_{N \backslash N_{k}} \in$ $\mathbb{R}^{n-n_{k}}$, and on a diagonal subspace $L^{D}$. In particular, the latter expresses the fact that under the stated conditions, an SWF is always a weighted utilitarian SWF if for all $k \in\{1, \ldots, m\}$, all individual utilities have the same value within every subgroup of individuals $N_{k}$.

It should be mentioned that if all subgroups $N_{k}, k \in\{1, \ldots, m\}$, are singletons, the hypothesis of Theorem 4.1 is equivalent to the condition of CUCinvariance for all $i \in N$. Also, combinations of different CUC scales with a common unit do not require separate study, since this amounts to supposing CUC-invariance for the entire society.

For combinations of CUC and CFC scales, without loss of generality it suffices to consider only the situation $N=N_{1}$ (CUC) $\cup N_{2}$ (CFC), which in turn is equivalent to the consideration of the combination of $n_{1}+1 \mathrm{CFC}$ scales with $n_{1}$ singleton subgroups.

Remark 4.2. As it was noted by Roberts (1980b), for the characterization of weighted utilitarianism, TSM invariance is enough. Thus, the conclusion of Theorem 4.1 concerning the linear form of the function $W$ on a diagonal subspace $L^{D}$ still holds if we consider a combination of TSF subgroup scales instead of that of CFC ones.

It is worth mentioning that if we consider the full continuum of different combinations of scales for the entire society between CM and CFC for all individuals, at "point" CM we have Sen's (1970) dictatorial result, between "points" CM and CUC there exists a dictatorial subgroup with a utilitarian rule (the result of Plata-Pérez (1994) which is a particular case of Theorem 3.1 for various combinations of independent among distinct subgroups CUC scales), at "point" CUC we arrive at a utilitarian rule for the entire society, between CUC and CFC we arrive at Theorem 4.1, and at CFC we have the result of Roberts (1980b), and Yanovskaya (1988). 


\section{Interval scales with a common zero.}

Next we show that every combination of interval scales with a common for the entire society zero leads to existence of a dictatorial subgroup. It is not hard to see that it suffices to consider only combinations of different CFC subgroup scales with a common zero.

Return back to the proof of Theorem 3.1 (see Appendix) and notice that for every combination of the subgroup scales $\Phi_{N_{k}}, k \in\{1, \ldots, m\}$, for which $W^{*}$ in the first step of the proof has to be dictatorial and for which $\tilde{c}$ is independent of $c$ in the second step, there exists a dictatorial subgroup among the subgroups $N_{k}$. Observe, that for the considered combinations these conditions are valid. Indeed, first, $W^{*}$ has to be invariant under strictly increasing affine transforms with a common constant term which results in $W^{*}$ to be dictatorial (see d'Aspremont (1985)). Second, every two transforms $\phi_{i}$ and $\phi_{j}$ relevant to subgroups $N_{k}$ and $N \backslash N_{k}$ are any two strictly increasing affine transforms with the only restriction that they have the same constant term, that combined with (3) obviously leads for $\tilde{c}$ to be independent of $c$. These observations together with the result of Roberts (1980b) for the CFC-characterization allow to prove

Theorem 4.3. Let $\left\{N_{k}\right\}_{k=1}^{m}$ be a partition of $N$ and let $W$ be a continuous, nondecreasing real-valued function defined on $\mathbb{R}^{n}$, that is invariant with respect to variables indexed by $N_{k}$ under CFC transforms that may be different for different $k$ but that have the same common constant term. Then there exists a unique integer $k \in\{1, \ldots, m\}$, such that for all $u \in \mathbb{R}^{n}$ the function $W(u)$ is determined only by the set of variables $N_{k}$ and has the form

$$
W(u)=\bar{u}_{N_{k}}+\theta\left(u_{N_{k}}-\bar{u}_{N_{k}}\right)
$$

where $\theta: \mathbb{R}^{n_{k}} \rightarrow \mathbb{R}^{1}$ is a continuous and homogeneous of the first degree function.

\section{Concluding remarks}

To conclude, it is worth noting that social orderings for problems with different utility scales for separate subgroups of individuals may be obtained by aggregating the individual utilities in two ways: either simultaneously over the entire society, or first within subgroups with identical information and then integrating the partially aggregated information. It is clear, that in the second case the second stage of the aggregation is equivalent to aggregation with a smaller number of individuals. However, there is no information available about the comparability of utilities for this smaller number of individuals, and so the situation is similar to that captured by the Arrovian conditions. Hence, every social ordering obtained by this way of aggregation is fully determined by the opinions of only one subgroup of individuals and it is in accordance with the measurement scales of its members' utilities. But we can not draw the same conclusion when we aggregate the individual utilities simultaneously over the entire society even for the combinations of independent among subgroups scales. We can show that 
this is true for all combinations of independent two-parameter subgroup scales with at most one one-parameter subgroup. However, if there are more than one subgroup of individuals with ratio or translation scale measurable utilities, then the social ordering may be determined by individual opinions of a few of them. Meanwhile, the combinations of interval subgroup scales with a common for the entire society zero give an example of combinations of dependent subgroup scales that lead to existence of a dictatorial subgroup.

\section{Appendix: Proofs}

Proof (of Lemma 3.2). Fix any $u \in \mathbb{R}^{n}$ and consider the level surface $\gamma(W(u)$ ) of $W$ containing $u$. Let $u^{\prime} \in \gamma(W(u)), u^{\prime} \neq u$. Assume that $u_{i_{0}}^{\prime} \neq u_{i_{0}}$. For any $\alpha>0$ consider an admissible transform $\phi=\left\{\phi_{i}\right\}_{i=1}^{n}$,

$$
\phi_{i}(t)=\phi_{i}\left(\alpha, u_{i_{0}} ; t\right)=\left\{\begin{array}{cc}
(1-\alpha) u_{i_{0}}+\alpha t, & i=i_{0} \\
t, & i \neq i_{0} .
\end{array}\right.
$$

By (3) for all $\alpha>0, W\left(\phi u^{\prime}\right)=W(\phi u)$. But for all $\alpha>0, \phi u=u$, and so for all $\alpha>0, \phi u^{\prime} \in \gamma(W(u))$. If $\alpha=1$, then $\phi u^{\prime}=u^{\prime}$. Therefore, the ray starting from the point $\tilde{u}=\left\{\begin{array}{l}u_{i_{0}}, i=i_{0} \\ u_{i}^{\prime}, i \neq i_{0}\end{array}\right.$ and proceeding parallel to the $i_{0}$-axis passes through the point $u^{\prime}$ and belongs to $\gamma(W(u))$. Using another transform $\psi=\left\{\psi_{i}\right\}_{i=1}^{n}$ admissible under any $\alpha>0$,

$$
\psi_{i}(t)=\psi_{i}\left(\alpha, u_{i_{0}}^{\prime} ; t\right)=\left\{\begin{array}{cc}
(1-\alpha) u_{i_{0}}^{\prime}+\alpha t, & i=i_{0}, \\
t, & i \neq i_{0}
\end{array}\right.
$$

we can check that the ray starting from $u^{\prime}$ and running parallel to the $i_{0}$-axis contains the point $\tilde{u}$ and belongs to $\gamma(W(u))$. Hence, a whole straight line parallel to the $i_{0}$-axis and containing $u^{\prime}$ lies completely in the level surface $\gamma(W(u))$.

Thus, if for the $i_{0}$-variable on some fixed level surface $\gamma$ of $W$, there exist $u^{\prime}, u^{\prime \prime} \in \gamma$, such that $u_{i_{0}}^{\prime} \neq u_{i_{0}}^{\prime \prime}$, then for every $u \in \gamma$ the whole straight line containing $u$ and parallel to the $i_{0}$-axis belongs to $\gamma$. For any function invariant under TSF transforms all level surfaces can be obtained from each other by a shift along the diagonal of the utility space. All variables of $W$ except RSM (RSF) ones, and in particular the $i_{0}$-variable, admit TSF transforms. With respect to RSM (RSF) variables (if such exist) $W$ is a homothetic function, and so, under RSM transforms applied to some $i$-variable, $i \neq i_{0}$, any straight line orthogonal to the $i$-axis transforms into a parallel one. Hence, if in any level surface of the function $W$, there exist two points with different $i_{0}$-components, then the function $W$ is independent of the $i_{0}$-variable. It follows immediately that either for all $u^{\prime} \in \gamma(W(u)) \quad u_{i_{0}}^{\prime}=u_{i_{0}}$ and, therefore, $W(u)=W\left(u_{i_{0}}\right)$, or $W(u)$ is independent of the $i_{0}$-variable.

Proof (of Theorem 3.1). First, consider the subdomain $L^{D}$ in which all variables indexed by the same subgroup of indices have the same value. In the 
obvious way the restriction of $W$ to $L^{D}$ can be identified with a continuous, nondecreasing real-valued function $W^{*}$ on $\mathbb{R}^{m}$. This function $W^{*}$ inherits the invariance property from $W$ and, hence, is invariant under CM transforms with respect to all variables $u_{k}, k \in\{1, \ldots, m\}$, corresponding to two-parameter type subgroups $N_{k}^{(2)}$. Because of Lemma 3.2, applying an induction argument to all $\mathrm{CM}$ variables sequentially, we can claim that either there exists a unique integer $k \in\{1, \ldots, m\}$, corresponding to $N_{k}^{(2)}$ such that the function $W^{*}$ has the form

$$
W^{*}(u)=W^{*}\left(u_{k}\right)
$$

for all $u \in \mathbb{R}^{m}$ or, for all $u \in \mathbb{R}^{m}, W^{*}$ depends only on variables $u_{k}$ relevant to one-parameter type subgroups $N_{k}$, and so, restricted to $L^{D}, W$ depends only on the variables indexed either by some $N_{k}^{(2)}, k \in\{1, \ldots, m\}$, or by the entire set of variables $N^{(1)}$. In what follows, the subgroup of variables determining $W$ on $L^{D}$ we denote by $N_{0}$.

Second, for all $i \notin N_{0}$, fix the variables $u_{i}$ to be $c$ and let $u_{N_{0}}$ be any arbitrary vector in $\mathbb{R}^{n_{0}}$. By (2), we have

$$
W\left(\hat{u}_{N_{0}}, c_{N \backslash N_{0}}\right) \geq W\left(u_{N_{0}}, c_{N \backslash N_{0}}\right) \geq W\left(\check{u}_{N_{0}}, c_{N \backslash N_{0}}\right) .
$$

Continuity then implies that there exists a real $\tilde{c}$ such that

$$
W\left(\tilde{c}_{N_{0}}, c_{N \backslash N_{0}}\right)=W\left(u_{N_{0}}, c_{N \backslash N_{0}}\right) .
$$

Because of (3) and the assumption that the transforms for the subgroups $N_{0}$ and $N \backslash N_{0}$ are independent, $\tilde{c}$ does not depend on $c$. Thus, the last equality, combined with the argument in step one, implies that $W$ only depends on the variables indexed by $N_{0}$ when $W$ is restricted to the subdomain of vectors in which the individuals not in group $N_{0}$ all have the same utility.

Third, we extend this result to the whole domain using a proof by contradiction. If $W$ does not only depend on the variables indexed by $N_{0}$, there must exist a vector of utilities $u_{N_{0}}$ for subgroup $N_{0}$ and two vectors of utilities $v_{N \backslash N_{0}}, w_{N \backslash N_{0}}$ for the individuals in $N \backslash N_{0}$ such that

$$
W\left(u_{N_{0}}, v_{N \backslash N_{0}}\right)>W\left(u_{N_{0}}, w_{N \backslash N_{0}}\right) .
$$

By (2), this inequality implies that

$$
W\left(u_{N_{0}}, \hat{v}_{N \backslash N_{0}}\right)>W\left(u_{N_{0}}, \check{w}_{N \backslash N_{0}}\right),
$$

and this contradicts the conclusion in step two. Hence, $W$ can only depend on the variables indexed by $N_{0}$.

Fourth, by the preceding argument, $W$ can be identified with a function $\bar{W}$ on $\mathbb{R}^{n_{0}}$. This function is continuous, nondecreasing, and is invariant under transforms of the class $\Phi^{(0)}$. The last statement in the theorem follows from these observations. 


\section{References}

Arrow, K.J. (1963): Social choice and individual values, (2nd ed.) New York: John Wiley and Sons.

Bossert, W., And J.A. Weymark (1996): "Utility in social choice," to appear in: Handbook of utility theory, volium 2: extensions, ed. by S. Barberá, P.J. Hammond, AND C. Seidl. Boston: Kluwer Academic Publishers.

D'Aspremont, C. (1985): "Axioms for social welfare orderings," in: Social goals and social organizations: essays in memory of Elisha Pazner, ed. by L. Hurwicz, D. Schmeidler, AND H. Sonnenschein. Cambridge: Cambridge University Press, 19-76.

D'Aspremont, C., And L. Gevers (1977): "Equity and the informational basis of collective choice," Review of Economic Studies, 44, 199-209.

Debreu, G. (1954): "Representation of a preference ordering by a numerical function," in: Decision processes, ed. by R.M. Thrall, C.H. Coombs, AND R.L. Davis. New York: John Wiley and Sons, 159-165.

HAMmOND, P.J. (1979): "Equity in two person situations: some consequences," Econometrika, 47, 1127-1135.

KAneKo, M., AND K. NAKAmura (1979): "The Nash social welfare function," Econometrica, 47, 423-435.

KhmelnitskayA, A.B. (1996): "Social choice problems with different scales of individual welfares measurement for different subgroups of individuals," in: Operations research proceedings 1995, ed. by P. Kleinschmidt, A. Bachem, U. Derigs, D. Fischer, U. Leopold-Wildburger, AND R. Möring. Berlin: Springer-Verlag, 252-257.

(1999): "Social welfare orderings for different subgroup utility scales," Discussion paper \#198, Center for Rationality and Interactive Decision Theory at The Hebrew University of Jerusalem.

Khmelnitskaya, A.B., And J.A. Weymark (2000): "Social choice with independent subgroup utility scales," Social Choice and Welfare, 17, 739748.

Phanzagl, J. (1971): Theory of measurement, (2nd ed.) Würzburg-Wien: Physica-Verlag.

PlatA-PÉrez L. (1994): "Aggregation and Sen's partial comparability," Unpublished manuscript, Departamento de Economía, Instituto Technológico Autónomo de México.

RoberTs, K.W.S. (1980): "Possibility theorems with interpersonally comparable welfare levels," Review of Economic Studies, 47, 409-420.

- (1980): "Interpersonal comparability and social choice theory," Review of Economic Studies, 47, 421-439.

Sen, A.K. (1970): Collective choice and social welfare. San Francisco: HoldenDay, and Edinburgh: Oliver and Boyd.

Tsui, K.-Y., AND J.A. WEYMARK (1997): "Social welfare orderings for ratioscale measurable utilities," Economic Theory, 10, 241-256.

YANOVSKAYA, E.B. (1988): "Social choice functions for different scales of individual preference measurement," (in Russian), Annals of VNIISI (All-Union Research Institute for System Studies, Moscow), 6, 64-76. 
(1989): "Group choice rules in problems with comparisons of individual preferences," Automation and Remote Control, 50, 822-830 (translated from the Russian original that appeared in Avtomatika $i$ Telemekhanika, 6, 129138). 